\title{
CREATION OF NANOROBOTS: BOTH STATE-OF-THE-SCIENCE AND STATE-OF-THE-ART
}

\author{
Parichehr Hassanzadeh, Fatemeh Atyabi, and Rassoul Dinarvand \\ Nanotechnology Research Center, Faculty of Pharmacy, Tehran University of Medical Sciences, \\ Tehran, Iran
}

Over the last decade, remarkable achievements in nanofabrication technology has led to the development of hybrid intelligent systems including the nanomechanical devices powered by the chemical energy sources or biomolecular motors. In this context, nanorobotics has emerged as a highly-advanced technology for designing the fully functional smart devices or robots at nano scale. Development of these highly-controlled and functional nanostructures for sensing, information processing, signaling, and actuation may provide remarkable breakthroughs in medicine such as the improved imaging or targeted therapeutic interventions. Besides the detection and destroying the toxic materials and ecosystem restoration, the stimuli-responsive nanorobots may be used for the diagnosis or treatment of cardiac disorders, traumatic injuries, diabetes, and bacterial or viral infections. These molecular tools with nanoscale resolution facilitates early diagnosis in cancer and precise localization of anticancer agents leading to the minimal side effects. Nanorobots may easily traverse the human body and repair the cells or assist an improper functioning organ. These tiny devices integrated with wireless locomotion, external or internal power supply, artificial intelligence, and smart sensors may also be used for targeted delivery of genes or drugs into the single cells or tissues, tele-operation, or patient monitoring. Indeed, development of the medical nanorobots with a wide range of capabilities is a proof of concept and art in modern science and a breakthrough in nanotechnology which has been highlighted in the present manuscript.

Biomed Rev 2016; 27: 19-26

Key words: smart devices, nanomachines, nanomanipulation, theranostic breakthroughs

Received 23 November 2016, accepted 8 December 2016.

Correspondence to Dr Parichehr Hassanzadeh, Nanotechnology Research Center, Faculty of Pharmacy,

Tehran University of Medical Sciences, Tehran, Iran Tel/Fax: +98 21 66959052; Cell phone: +98 9121887745

E-mail: p-hassanzadeh@razi.tums.ac.ir 


\section{INTRODUCTION}

The promising advances in miniaturizing approaches including the construction of microscopic biomechanical devices have led to the development of highly sophisticated nanoscale platforms which may revolutionize the current theranostic strategies. The emerging technology of nanorobotics; creating the robot nanomachines with innovative hardware architecture, artificial intelligence and sensors (temperature, force, light, or position sensors) and accurate targeting ability has been one of the most important discoveries in nanotechnology (1-3). Application of various scientific disciplines including the nanotechnology, biomedical, chemical, and mechanical engineering as well as physics, biology, and mathematics is required for the development of these smart tiny machines which provide the possibility of precise interactions with nanoscale objects, onboard computation, power transmission, sensing, or nanomanipulation (4). Integration of biomaterials, nanoelectronics, and photolithography provides the possibility to design the nanorobots for theranostic purposes, drug delivery, advanced medical instrumentation, patch clamping, or surgery on the individual cells.

Nanorobots may also be used for tissue repair. Following the tissue injury, white blood cells are recruited to the affected area. Because of its small size, a nanorobot is able to attach to the surface of white blood cell and moves towards the site of injury for being involved in the tissue repair process through a complex mechanism which may involve the vasodilation or activation of cell surface receptors $(1,2,5,6)$. Diffusion and interaction of nanorobots as the artificially-fabricated programmable objects with distributive intelligence and evolutionary behavior in the human body may be controlled by internal and/or external mechanisms (7). In order to better understand the mechanism/s of the interaction of nanorobots with an living organism, advanced simulation techniques should be applied $(8,9)$. Indeed, nanorobotic treatment associated with modeling approaches may revolutionize the current treatment strategies in medicine.

The size of an assembled biorobot may be within the range of 0.5-0.8 $\mu \mathrm{m}$. Based on the diameter of a capillary (5-10 $\mu \mathrm{m})$, these types of microrobots may be easily navigated in the blood stream. It is worth mentioning that the macro- or microrobots which move with nanoscale precision may also be considered as nanorobots. The optimal shape of a nanorobot usually depends on its task and the environment in which it should operate $(10,11)$. Three-dimensional (3D) printing at nanoscale using a laser etching machine may be used for manufacturing of nanorobots. 3D printing elevates the process accuracy and improves the functionality of nanorobot segments (10). The photoactive resins which are used in $3 \mathrm{D}$ printers, may be hardened by a focused laser beam. Then, the laser beam is guided through the resin and a hardened line of a solid polymer at nanoscale is created leading to the production of tiny ( $<100 \mathrm{~nm}) 3 \mathrm{D}$ objects (10). The body of a nanorobot may be constructed from carbon-based materials such as fullerene nanocomposites or carbon nanotubes because of their unique properties such as high biocompatibility, strength, and thermoelectrical conductivity as well as the biosensing ability, absorbing near-infrared radiation, and providing sustained therapeutic effects (12-16). Meanwhile, the chemically inert diamond is usually preferred for exterior coating of the medical nanorobots as the carbon atoms in diamondoid structures provide super-smooth surfaces leading to the reduced risk of immunological reactions $(10,17)$.

Regarding the movement of nanorobots, synthetic molecular motors may be attached to the surface of these nanomachines to control their motion. Indeed, the mechanism of movement of a nanorobot in a liquid environment remains as a major concern in the design process. During the navigating in blood stream, these high-performance nanoscale platforms should face a smooth path. Furthermore, appropriate strategies should take into account to prevent any damage to healthy cells in the case of oncotherapy. Upon the disease detection, nanorobots should be highly responsive and move their tentacles towards the target cells. This, necessitates the embedding of a microcomputer including a miniature processor within the architecture of nanorobot $(5,10,11)$. In general, activities of kinesin, dynein, and myosin protein families which are critically involved in the biological locomotion (18) are simulated for the movement of nanorobots. In the biological or medical nanorobots, the microorganisms such as Salmonella typhimurium or Escherichia coli may be used in which the flagella (a biological motor) facilitates the movement of bacteria in a liquid environment by the energy obtained from the hydrolysis of adenosine triphosphate and overcomes the opposing forces of viscous drag on the body of bacteria. The motion of these nanorobots may be controlled by an electromagnetic field (19, $20)$. Besides the application of glucose biofuel in the creation of bionanomachine, the moving parts of the synthetic nanomachines may consist of the semiconductor nanowires, metal, or carbon nanotubes which are driven by the electrical energy (21). A nanorotor containing the suspended carbon nanotube beams and an attached silicon nanoplate between the two 
electrodes converts the electrical energy into the rotary motion of the plate. These tiny rotors may be utilized as the nanostirrers in order to agitate the liquids particularly in the confined spaces (22). For the rotary or linear type of motion, a metal nanowire may be applied which is suspended in a droplet of water between the microelectrodes with particular geometry. The degree of nanowire rotation, rotation speed, and velocity are under the electrical control $(23,24)$. For nanolocomotion, nanocrystal linear nanomotors have also been designed using the indium nanocrystals and carbon nanotubes (25).

In order to perform the macroscopic or microscopic tasks, a large number of nanorobots should work together because of their microscopic size. In this respect, ultrasonic sensors are usually attached around the body of a nanorobot to prevent the possible collision with each other or other cells (26). Upon the completion of nanorobot task in the human body, they may be removed through the usual excretory channels or scavenger systems $(27,28)$.

\section{APPLICATIONS OF NANOROBOTS}

Besides the detection or measurement of toxic chemicals in the environment, nanorobots may be used for health care. In this respect, robots at micro or nano scale have been designed for the elimination or treatment of medical problems including the blood clots, scare tissue, arteriosclerosis, tumors, kidney stones, or different types of infections (5-7). Indeed, using the complex nanomechanical systems facilitates the biomedical instrumentation, tele-operation, targeted drug delivery, or monitoring of the metabolic disorders such as diabetes (5, 29). In this respect, nanorobots have received a large number of patents (30). Nanorobots may be programmed to perform the biological tasks even at the cellular level. For instance, they may be injected into the blood to attack the cancer cells or viruses. In order to attract the nanorobots towards the cancerous cells, folate materials may be attached on the body of nanorobots which are powered by flagella motors; a set of rotary motors which provide a movement speed of $25 \mu \mathrm{m} / \mathrm{s}$ (5, 31). A similar approach may be taken to deliver the medications against the human immunodeficiency virus (32).

In the case of arteriosclerotic plaques, medical nanorobots which swim in the bloodstream are able to widen the affected blood vessels and prevent the heart attacks (33). In the stenotic blood vessels specially the coronary arteries, these nanodevices may be used for the treatment of atherosclerotic lesions (34). Medical nanorobots may also be used for monitoring the glucose levels in diabetic patients or other nutrient concentrations in the body (5). Recently, a smart nanomachinebased mouthwash has been designed to detect and destroy the pathogenic bacteria without affecting the harmless flora of the mouth. This mouthwash is able to identify and remove the plaques or food particles which are beyond the access of the fibers of floss or toothbrush bristles (35). Moreover, a cream containing the nanorobots has been produced for the treatment of skin diseases through the removal of excess oils or dead skin cells, deep pore cleaning, or applying natural moisturizing compounds (36). In this sense, nanorobtos may be able to prevent the aging (37).

As aforementioned, integration of the medical nanorobot architecture into the living system might be of theranostic significance. Magnetic resonance imaging (MRI)-guided nanorobots or nanomotors operating in the living organism may be applied for early disease diagnosis which is of critical importance in cancer. These controllable nanomachines are able to algorithmically respond to input forces and information $(38,39)$. Indeed, the suitability of MRI approach is because of the availability of MRI devices, non-invasiveness, capability to provide accurate localization of the magnetic particles, or track and actuate the nanoparticles simultaneously $(40,41)$. In MRIguided nanorobotic systems, MRI scanner is used to induce an external driving force for guidance of the nanoparticles towards their target. In this context, chemical modifications of the nanoparticles and application of the navigation techniques might result in a highly-controlled and targeted treatment (3841). Regarding the development of MRI-guided nanorobotic drug delivery, the MRI-guided targeted drug delivery system has been designed which is capable of simultaneous imaging and propulsion leading to the real-time control of drug targeting process (38). In general, the MRI-based nanorobotic architecture includes; $(i)$ a nanocapsule which is the most important part of the system. The main body of the nanocapsule is constituted of a nanocarrier which is used for drug loading. Polymer chains or biomolecules are usually attached to the nanocarrier to enhance the biocompatibility and solubility and provide sustained circulation of the nanocapsule, (ii) magnetic nanoparticles which induce propulsion forces, (iii) drug molecules which may be chemically attached onto the surface of the carrier or loaded within the nanocarrier, (iv) sensing elements including the biomolecules which sense the environmental conditions surrounding the nanocapsule, avoid the immunogenic reactions, trigger drug release, or target the specific molecules, $(v)$ MRI propulsion and tracking units, vi) high-level controller (real-time control algorithm), and (vii) 
a distributed computer system, 3D-visualization, and process supervision tools including the software-based upgrading of the MRI system. In this context, the endovascular navigation may be performed via the integration of MRI tracking events and propulsion system with real-time control algorithms (3845 ). Besides their therapeutic potential, some nanoparticles exhibit optical properties which make them suitable candidates for bio-imaging. These types of nanoparticles may be used in MRI-guided nanorobotic systems. Quantum dots, gold nanoparticles, polymer micelles, liposome nanoparticles, metallic nanoshells, and carbon nanotubes may be used as the carrier module in MRI-guided nanorobotic systems. The surface of these nanomaterials may be chemically modified with biomolecules or copolymers to improve their biocompatibility, solubility, stability, and sensing ability (43-45). In general, magnetic targeting is a multiscale process which involves various physical domains including the fluid dynamics and magnetic fields that necessitates the application of modeling approaches (8, 9, 38-45). Furthermore, appropriate control algorithms should be applied for stable and automatic control of nanocarriers against the drag wall forces and blood flow as the environment perturbations $(6,45)$. In cancer therapy, nanorobotic capsules should be guided from the injection point to the tumor area. In this respect, a 3D navigation path into the blood vessels should be provided using 3D MRI imaging which significantly elevates the targeting accuracy and therapeutic efficiency of the anticancer agents because of the trajectory tracking ability $(11,27)$.

\section{NANOROBOTS: SMART DRUG CARRIERS}

The incidence of adverse drug reactions due to the improper delivery methods has remained as a major challenge in medicine. Over the last decade, nanotechnology has provided a wide range of novel approaches and techniques to optimize drug delivery methods. In this respect, nanorobots have been represented as promising devices for efficient targeted drug delivery that might be of great significance particularly in chemotherapy $(5,11,27,44)$. In the early stages of development, the chemical biosensors-embedded nanorobots may be used to detect the tumor cells. The integrated sensors are able to detect the E-cadherin molecules or acoustic signals $(27,44,46,47)$. Regarding the controlled drug delivery and superior targeting ability, the wire-shaped magnetoelectric nanorobots have been designed which may be wirelessly steered towards the target by rotating the magnetic fields and then release their cargo (e.g. an anti-cancer drug). In order to fabricate magnetoelectrically-assisted nanodevices for drug release, the hybrid magnetoelectric composite nanowires including the piezoelectric shells and magnetostrictive cores are used. Application of the biphasic core-shell configuration provides more flexibility in the development process (48). As an external power source, magnetic field is used because of its high biocompatibility and accuracy to control the locomotion of magnetic structures (49). Based on the numerous adverse effects of anticancer agents, development of the externally-controlled nanocarriers which are able to release the chemotherapeutics on-site might be of great therapeutic value. In this sense, magnetoelectric nanowire robots in which the magnetic fields precisely control the locomotion of these nanorobots have been represented as the valuable biocompatible nanodevices for targeted drug delivery. For enhancement of drug loading, nanowires are usually pretreated with polydopamine in order to bond the hydroxyl and amine groups on their surface (surface functionalization). This, results in the adsorption of drug onto the surface of core-shell nanorobot via the chemical interactions between polydopamine and drug molecules $(50,51)$. Upon the exposure of drug delivery vehicle to an alternating magnetic field, the chemical bindings between polydopamine and drug are broken leading to the drug release from the nanowire (52). In this respect, application of different magnetic fields has been suggested as a promising approach for the development of nanorobots for controlled drug delivery (52). For targeted drug delivery in the cardiovascular system, an integrated nanostructure composed of nanosensors and nanoactuatros has been used for the navigation of ferromagnetic microcapsules $(6,19,40$, $41,43)$. This, might be of great theranostic significance due to the improved propulsion and diagnostics in MRI which facilitates the localized treatment.

A drug or biomolecule entrapped within a nanorobot may be released at very precise points by manipulating the physiological conditions. In these nanomamchines, the release process at the target site is usually triggered by the alterations of temperature or $\mathrm{pH}$. Since the unexpected changes in the physiological conditions may occur, therefore, development of the externally-triggered nanorobots might provide more suitable controlled delivery strategy (52).

In recent years, DNA nanorobots using a method called DNA origami with capability of responding to the external stimulus and delivery of cellular compatible message have been produced. The switch actuator of this type of nanorobot is triggered by the target external nucleic acid which interacts 
with the complementary nucleic on nanorobot. Besides the biosensing, DNA nanorobots may be used for intelligent delivery of biological activators $(5,53)$. For preparation of DNA nanorobots, the sequence of DNA strands may be initially defined using the software programs and after synthesis and mixing together, the complex 3D shapes capable to entrap the drugs such as the anticancer agent are obtained (54). Furthermore, DNA nanorobots have been shown to trigger apoptosis in leukemia cells in vitro (55).

Over the last decade, nerve growth factor (NGF) and endocannabinoids have attracted a growing interest due their metabotrophic or neurotrophic effects. They are implicated in the neuroprotective processes and mechanisms of action of a wide variety of therapeutic agents (56-62). However, poor solubility, rapid degradation, or non-specific distribution of endocannabinoids or $\operatorname{NGF}(63,64)$ may limit the efficiency of these modulators. Based on the unique properties of smart nanorobots including their capability for controlled targeted delivery of drugs or biomolecules, entrapment of NGF or endocannabinoids into these nanomachines might provide prolonged therapeutic effects.

\section{LIMITATIONS AND HOPES IN NANOROBOT DESIGN}

Navigation is a fundamental necessity for cell-specific actions and communications of nanorobots. Meanwhile, navigation of nanorobots through a fluid phase appears as a major challenge due to the continuous collisions within the body fluids that may result in the deviation of a nanorobot from its specific target (2, $4,5)$. In this respect, novel techniques have been developed to assist these nanodevices to swim towards their destination. For instance, novel generation of motors based on the nanoscale building blocks have been introduced which are able to move through a fluid environment and perform particular tasks (1, 18, 20). However, the swimming behavior of nanorobots may be dramatically affected by the components, viscosity, or temperature of the biological fluids (65). In this respect, nanorobots have been designed which are magnetically guided towards the injury site or their movement may be facilitated using the micro-motors powered by the chemical reactions within the body $(19,44,45,48)$. Using an oscillating magnetic field, nanorobots containing the magnetic metal nanowires with S-shaped motion have also been designed. Interestingly, the magnetic field is able to dictate the direction of nanorobot movement towards the target $(39,41,43)$.

Providing an efficient energy source which is essential for the mechanical movements or chemical transformations may be another challenge in nanorobot design. Nanorobot should be able to metabolize the oxygen and glucose in vivo or an external power should be supplied. Usually, the same external power source is utilized for both delivery of therapeutic agent and locomotion that may raise concerns due to the possible interference between these two tasks of nanorobots. The external control problems remain as further concerns when multiple nanomachines are used (19, 20, 42). Heat dissipation, specially in the case of the deployment of a large number of nanorobots, and communication of nanorobots which is necessary to perform the complex activities, monitor the collective task progress, and receive or transmit the messages, are other challenges which should be addressed $(1,2)$. Altogether, special attention should be paid to the precise fabrication and control strategies, advanced simulation techniques, wireless communication, and size reduction in order to obtain more efficient and fully functional nanorobots for long-term benefits.

\section{CONCLUSION}

Over the last decade, nanotechnology-based treatment strategies have shown enormous potential against the ineffectiveness of some conventional therapeutic agents due to their poor solubility or lack of target specificity. Miniaturization through the nanotechnology has provided the possibility to construct devices and materials at nanoscale for being used in advanced theranostic settings. Medical nanorobots are the beneficial proof-of-concept integrated nanomachines with ability for early detection and destroying the pathogens, smart drug delivery, and individual cell surgery in vivo that might be of critical significance. As an alternative to chemotherapy, application of nanorobots may result in the detection and destroying of cancer cells without affecting the healthy cells. Despite the potential limitations, nanorobotic treatment appears to be a major part of the future medicine. In fact, manipulation of materials at molecular level and controlling their properties including the automation are serious challenges in the development of bionanorobots. Meanwhile, as Nikola Tesla said "The scientific man does not aim at an immediate result. He does not expect that his advanced ideas will be readily taken up. His work is like that of the planter-for the future. His duty is to lay the foundation for those who are to come, and point the way".

Conflict of interest: the authors declare no conflict of interest. 


\section{REFERENCES}

1. Cavalcanti A, Shirinzadeh B, Freitas Jr. RA, Kretly LC. Medical nanorobot architecture based on nanobioelectronics. Recent Pat Nanotechnol 2007; 1: 1-10. PMID:19076015.

2. Cavalcanti A, Shirinzadeh B, Zhang M, Kretly LC. Nanorobot hardware architecture for medical defense. Sensors 2008; 8: 2932-2958. DOI:10.3390/s8052932.

3. Hassanzadeh P. New perspectives in biosensor technology. Gastroenterol Hepatol Bed Bench 2010; 3: 105-107.

4. Murphy D, Challacombe B, Nedas T, Elhage O, Althoefer $\mathrm{K}$, Seneviratne L, et al. Equipment and technology in robotics. Arch Esp Urol 2007; 60: 349-354.

5. Patel GM, Patel GC, Patel RB, Patel JK, Patel M. Nanorobot: A versatile tool in nanomedicine. J Drug Target 2006; 14: 63-67. DOI:10.1080/10611860600612862.

6. Ohki T, Ouriel K, Silveira PG, Katzen B, White R, Criado $\mathrm{F}$, et $a$ l. Initial results of wireless pressure sensing for endovascular aneurysm repair: the APEX trial-acute pressure measurement to confirm aneurysm sac exclusion. J Vasc Surg 2007; 45: 236-242. DOI:10.1016/j.jvs.2006.09.060.

7. Benenson Y, Gil B, Ben-Dor U, Adar R, Shapiro E. An autonomous molecular computer for logical control of gene expression. Nature 2004; 429: 423-429. DOI: 10.1038/ nature 02551 .

8. Dubey A, Sharma G, Mavroidis C, Tomassone MS, Nikitczuk K, Yarmushc ML. Computational studies of viral protein nano-actuators. J Comp Theor Nanosci 2004; 1: $18-28$.

9. Hassanzadeh P. Computational modelling: moonlighting on the neuroscience and medicine. Biome Rev 2013; 24: 25-31.

10. Ignatyev MB. Necessary and sufficient conditions of nanorobot synthesis. Doklady Mathematics 2010; 82: 671-675. DOI:10.1134/S1064562410040435.

11. Hill C, Amodeo A, Joseph JV, Patel HR. Nano- and microrobotics: How far is the reality? Expert Rev Anticancer Ther 2008; 8: 1891-1897.DOI: 10.1586/14737140.8.12.1891.

12. Hassanzadeh P, Arbabi E, Atyabi F, Dinarvand R. Carbon nanotube-anandamide complex exhibits sustained protective effects in an in vitro model of stroke. Physiol Pharmacol 2016; 20: 12-23. http://phypha.ir/ppj/article1-1155-en.html

13. Hassanzadeh P, Arbabi E, Rostami F, Atyabi F, Dinarvand R. Carbon nanotubes prolong the regulatory action of nerve growth factor on the endocannabinoid signaling.
Physiol Pharmacol 2015; 19: 167-176. http://phypha.ir/ ppj/article-1-1112-en.html

14. Hassanzadeh P, Arbabi E, Atyabi F, Dinarvand R. Application of carbon nanotubes as the carriers of the cannabinoid, 2-arachidonoylglycerol: Towards a novel treatment strategy in colitis. Life Sci 2016; DOI:10.1016/j.lfs.2016.11.015

15. Hassanzadeh P, Arbabi E, Atyabi F, Dinarvand R. Nerve growth factor-carbon nanotube complex exerts prolonged protective effects in an in vitro model of ischemic stroke. Life Sci 2016; DOI: 10.1016/j.1fs.2016.11.029.

16. Hassanzadeh P, Arbabi E, Atyabi F, Dinarvand R. Application of carbon nanotubes for controlled release of growth factors or endocannabinoids: A breakthrough in biomedicine. Biomed Rev 2016; 27: 19-27.

17. T Kubik, K Bogunia, M Sugisaka. Nanotechnology on duty in medical applications. Curr Pharm Biotechnol 2005;6:17-33. PMID:15727553

18. Mallik R, Gross SP. Molecular motors: strategies to get along. Curr Biol 2004; 14: R971-R982. DOI 10.1016/j. cub.2004.10.046.

19. Martel S, Mohammadi M, Felfoul O, Zhao Lu, Pouponneau P. Flagellated magnetotactic bacteria as controlled MRI-trackable propulsion and steering systems for medical nanorobots operating in the human microvasculature. Int $J$ Robot Res 2009; 28: 571-582. DOI: 10.1177/0278364908100924.

20. Behkam B, Sitti M. Bacterial flagella-based propulsion and on/off motion control of microscale objects. Appl Phys Lett 2007; 90: 1-3.

21. Ozin GA, Manners I, Fournier-Bidoz S, Arsenault A. Dream nanomachines. Adv Mater 2005; 17: 3011-3018. DOI: 10.1002/adma.200501767.

22. Fennimore AM, Yuzvinsky TD, Han WQ, Fuhrer MS, Cumings J, Zettl A. Rotational actuators based on carbon nanotubes. Nature 2003; 424: 408-410. DOI:10.1038/ nature 01823

23. Fan DL, Zhu FQ, Cammarata RC, Chien CL. Efficiency of assembling of nanowires in suspension by ac electric fields. Appl Phys Lett 2006; 89: 10.1063/1.2397384. DOI: 10.1063/1.2397384

24. Fan DL, Zhu FQ, Cammarata RC, Chien CL. Controllable high-speed rotation of nanowires. Phys Rev Lett 2005; 94 : 247208. DOI: 10.1103/PhysRevLett.94.247208.

25. Regan BC, Aloni S, Jensen K, Ritchie RO, Zettl A. Nanocrystal-powered nanomotor. Nano Lett 2005; 5: 1730-1733. DOI: 10.1021/n10510659. 
26. Cavalcanti A, Freitas Jr. RA. Autonomous multi-robot sensor-based cooperation for nanomedicine. Int $J$ Nonlinear Sci Numer Simul 2002; 3: 743-746.

27. Martel S, Felfoul O, Mohammadi M, Mathieu JB. Interventional procedure based on nanorobots propelled and steered by flagellated magnetotactic bacteria for direct targeting of tumors in the human body. Conf Proc IEEE Eng Med Biol Soc 2008; 2008: 2497-500. DOI: 10.1109/ IEMBS.2008.4649707.

28. Curtis ASG, Dalby M, Gadegaard N. Cell signaling arising from nanotopography: implications for nanomedical devices. Nanomedicine 2006; 1: 67-72. doi: $10.2217 / 17435889.1 .1 .67$

29. Couvreur P, Vauthier C. Nanotechnology: intelligent design to treat complex disease. Pharm Res 2006; 23: 1417-1450. DOI:10.1007/s11095-006-0284-8

30. Celikten A; Cetin A. Recent advances, issues and patents on medical nanorobots. Rec Pat Eng 2016; 10: 28-35.

31. Balasubramanian S, Kagan D, Jack HCM, Campuzano $\mathrm{S}$, Lobo-Castañon MJ, Lim N, et al. Micromachineenabled capture and isolation of cancer cells in complex media. Angew Chem Int Ed Engl 2011; 50: 4161-4164. DOI:10.1002/anie.201100115.

32. Patel RR. Nanorobotics ideas in nanomedicine. Asian $J$ Pharm Sci Res 2013; 3: 15-22. ISSN 2249-4898.

33. Saadeh Y, Vyas D. Nanorobotic applications in medicine: Current proposals and designs. Am J Robot Surg 2014; 1: 4-11. PMID: 26361635

34. Kubik T, Bogunia-Kubik K, Sugisaka M. Nanotechnology on duty in medical applications. Curr Pharmaceut Biotechnol 2005; 6: 17-33. DOI: 10.2174/1389201053167248.

35. Sujatha V, Suresh M, Mahalaxmi S. Nanorobotics-A futuristic approach. SRM Univ J Dent Sci 2010; 1:86-80. https://www.researchgate.net/publication/216720568.

36. Mitthra S, Karthick A, Anuradha B, Mensudar R, Sadhana KR, Varshini GN. Nanorobots - A small wonder. Biosci Biotech Res 2016; 13: 2131-2134.

37. Freitas RA. The future of nanofabrication and molecular scale devices in nanomedicine. Stud Health Technol Inform 2002; 80: 45-59. PMID:12026137.

38. Vartholomeos P, Fruchard M, Ferreira A, Mavroidis C. MRI-guided nanorobotic systems for therapeutic and diagnostic applications. Annu Rev Biomed Eng 2011; 13: 157-184. DOI: 10.1146/annurev-bioeng-071910-124724.

39. Abbott JJ, Ergeneman O, Kummer MP, Hirt AM,
Nelson BJ. Modeling magnetic torque and force for controlled manipulation of soft-magnetic bodies. IEEE Trans Rob 2007; 23: 1247-1252. DOI: 10.1109/ AIM.2007.4412546.

40. Martel S, Mathieu JB, Felfoul O, Chanu A, Aboussouan $\mathrm{E}$, Tamaz S, et al. A computer-assisted protocol for endovascular target interventions using a clinical MRI system for controlling untethered microdevices and future nanorobots. Comput Aided Surg 2008; 13: 340-352. DOI:10.3109/10929080802551274.

41. Mathieu JB, Martel S. Magnetic microparticle steering within the constraints of an MRI system: proof of concept of a novel targeting approach. Biomed Microdev 2007; 9: 801-808. DOI: 10.1007/s10544-007-9092-0

42. Behkam B, Sitti M. Design methodology for biomimetic propulsion of miniature swimming robots. ASME J Dyn Sys Measur Control 2006; 128: 36-43. DOI: 10.1115/1.2171439

43. Carpi F, Pappone C. Magnetic manouvering of endoscopic capsules by means of a robotic navigation system. IEEE Trans Biomed Eng 2008; 62: 546-549. DOI: 10.1109/ TBME.2009.2013336.

44. Jurgons R, Seliger C, Trahms AHL, Odenbach S, Alexiou C. Drug loaded magnetic nanoparticles for cancer therapy. J Phys Condens Matter 2006; 18: 2893-2902.

45. Sun C, Lee JSH, Zhang M. Magnetic nanoparticles in MRI imaging and drug delivery. Adv Drug Deliv Rev 2008; 60: 1252-1265. DOI: 10.1016/j.addr.2008.03.018.

46. Cerofolini G, Amato P, Asserini M, Mauri G. A surveillance system for early-stage diagnosis of endogenous diseases by swarms of nanobots. Adv Sci Lett 2010; 3: 345-352. DOI: $10.1166 /$ asl.2010.1138

47. Hassanzadeh P, Fullwood I, Sothi S, Aldulaimi D. Cancer nanotechnology. Gastroenterol Hepatol Bed Bench 2011; 4: 63-69. PMCID: PMC4017405.

48. Chen XZ, Hoop M, Shamsudhin N, Huang T, Özkale B, Li Q, et al. Hybrid magnetoelectric nanowires for nanorobotic applications: fabrication, magnetoelectric coupling, and magnetically assisted in vitro targeted drug delivery. Adv Mat 2017; 1605458.DOI: 10.1002/ adma.201605458.

49. Kim S, Qiu F, Kim S, Ghanbari A, Moon C, Zhang L, et al. Fabrication and characterization of magnetic microrobots for three-dimensional cell culture and targeted transportation. Adv Mater 2013; 1-6. DOI: 10.1002/ adma.201301484 
50. Lee YS, Bae JY, Koo HY, Lee YB, Choi WS. A remotecontrolled generation of gold@polydopamine (core@ shell) nanoparticles via physical-chemical stimuli of polydopamine/gold composites. Sci Rep 2016; 6: 22650. DOI: $10.1038 /$ srep22650

51. Qian W, Yu DG, Li Y, Liao YZ, Xia Wang X, Wa L. Dual drug release electrospun core-shell banofibers with tunable dose in the second phase. Int J Mol Sci 2014; 15: 774-786. doi: 10.3390/ijms 15010774

52. Somanna MB. Nanobots: The future of medical treatments. Int J Sci Tech Res 2015; 4: 276-278. ISSN 2277 8616

53. Torelli E, Marini M, Palmano S, Piantanida L, Polano C, Scarpellini A, et al. A DNA origami nanorobot controlled by nucleic acid hybridization. Small 2014; 10: 2918-2926. DOI: $10.1002 /$ smll.201400245

54. Rothemund PW. Folding DNA to create nanoscale shapes and patterns. Nature 2006; 440: 297-302. DOI: 10.1038/ nature04586.

55. Douglas SM, Bachelet I, Church GM. A logic-gated nanorobot for targeted transport of molecular payloads. Science 2012; 335: 831-834. DOI: 10.1126/science. 1214081.

56. Yanev S, Aloe L, Fiore M, Chaldakov GN. Neurotrophic and metabotrophic potential of nerve growth factor and brain-derived neurotrophic factor: Linking cardiometabolic and neuropsychiatric diseases. World J Pharmacol 2013; 2: 92-99. DOI: 10.5497/wjp.v2.i4.92

57. Hassanzadeh $P$, Hassanzadeh A. Involvement of the neurotrophin and cannabinoid systems in the mechanisms of action of neurokinin receptor antagonists. Eur Neuropsychopharmacol 2011; 21: 905-917. DOI:10.1016/j. euroneuro.2011.01.002.
58. Hassanzadeh $\mathrm{P}$, Rahimpour S. The cannabinergic system is implicated in the upregulation of central NGF protein by psychotropic drugs. Psychopharmacology 2011; 215: 129-141. DOI:10.1007/s00213-010-2120-4.

59. Hassanzadeh P, Hassanzadeh A. Effects of different psychotropic agents on the central nerve growth factor protein. Iran J Basic Med Sci 2010; 13: 202-209.

60. Hassanzadeh P, Hassanzadeh A. Implication of NGF and endocannabinoid signalling in the mechanism of action of sesamol: a multi-target natural compound with therapeutic potential. Psychopharmacology 2013; 229: 571-578. DOI:10.1007/s00213-013-3111-z.

61. Hassanzadeh P, Hassanzadeh A. The $\mathrm{CB}_{1}$ receptor-mediated endocannabinoid signaling and NGF: The novel targets of curcumin. Neurochem Res 2012; 37: 1112-1120. DOI:10.1007/s11064-012-0716-2.

62. Hassanzadeh P, Arbabi E, Atyabi F, Dinarvand R. The endocannabinoid system and NGF are involved in the mechanism of action of resveratrol: a multi-target nutraceutical with therapeutic potential in neuropsychiatric disorders. Psychopharmacology 2016; 233: 1087-1096. DOI:10.1007/s00213-015-4188-3.

63. Jarho P, Urtti A, Pate DW, Suhonen P, Jarvinen T. Increase in aqueous solubility, stability and in vitro corneal permeability of anandamide by hydroxypropyl- $\beta$-cyclodextrin. Int J Pharm 1996; 137: 209-216. SICI:0378-5173.64.

64. Lindsay RM. Neurotrophic growth factors and neurodegenerative diseases: therapeutic potential of the neurotrophins and ciliary neurotrophic factor. Neurobiol Aging 1994; 15: 249-251. PMID:7838303.

65. Sharma NN, Mittal RK. Nanorobot movement: Challenges and biologically inspired solutions. Int J Smart Sens Intel Sys 2008; 1: 87-109. 